UDC: 519.21

\title{
Isotropic Multidimensional Catalytic Branching Random Walk with Regularly Varying Tails
}

\author{
E. Vl. Bulinskaya \\ Novosibirsk State University, \\ 1 Pirogova st., Novosibirsk, Russia, 630090 \\ E-mail: bulinskaya@mech.math.msu.su
}

Received 30.05.2019.

Accepted for publication 14.11.2019.

\begin{abstract}
The study completes a series of the author's works devoted to the spread of particles population in supercritical catalytic branching random walk (CBRW) on a multidimensional lattice. The CBRW model describes the evolution of a system of particles combining their random movement with branching (reproduction and death) which only occurs at fixed points of the lattice. The set of such catalytic points is assumed to be finite and arbitrary. In the supercritical regime the size of population, initiated by a parent particle, increases exponentially with positive probability. The rate of the spread depends essentially on the distribution tails of the random walk jump. If the jump distribution has "light tails", the "population front", formed by the particles most distant from the origin, moves linearly in time and the limiting shape of the front is a convex surface. When the random walk jump has independent coordinates with a semiexponential distribution, the population spreads with a power rate in time and the limiting shape of the front is a star-shape nonconvex surface. So far, for regularly varying tails ("heavy" tails), we have considered the problem of scaled front propagation assuming independence of components of the random walk jump. Now, without this hypothesis, we examine an "isotropic" case, when the rate of decay of the jumps distribution in different directions is given by the same regularly varying function. We specify the probability that, for time going to infinity, the limiting random set formed by appropriately scaled positions of population particles belongs to a set $B$ containing the origin with its neighborhood, in $\mathbb{R}^{d}$. In contrast to the previous results, the random cloud of particles with normalized positions in the time limit will not concentrate on coordinate axes with probability one.
\end{abstract}

Keywords: catalytic branching random walk, spread of population

Citation: Computer Research and Modeling, 2019, vol. 11, no. 6, pp. 1033-1039.

The work is supported by Russian Science Foundation under grant 17-11-01173 and is fulfilled at Novosibirsk State University. The author is Associate Professor of the Lomonosov Moscow State University. 


\section{Introduction}

The spread of a population of particles (genes, bacteria, individuals, etc.) has attracted the attention of researchers for a long time. Nowadays such phenomena can be described with the help of probabilistic mathematical models called branching random walks (BRW), combining particles splitting and their movement in space (see, e.g., [Bhattacharya et al., 2018] and [Shi, 2015]). If the particles' evolution obeys the same laws in any spatial area, BRW is space-homogeneous. We are interested in a nonhomogeneous BRW called catalytic (CBRW), see, e.g., [Bulinskaya, 2015a]. In CBRW the particles may produce offspring only in the presence of catalysts taking fixed positions on a multidimensional lattice $\mathbb{Z}^{d}, d \in \mathbb{N}$. Outside the catalysts the particles perform a random walk without branching. We study the propagation of the most distant (from the origin) particles at time $t$ forming the population "front", bordering, in a sense, the inhabited area and the empty environment, as $t \rightarrow \infty$. Having properly normalized the particles' positions at time $t$ and letting $t$ grow indefinitely, we specify the probability that the limiting random set formed by the scaled positions of particles belongs to a set $B$ in $\mathbb{R}^{d}$, containing the origin with its neighborhood. The proofs combine analysis of nonlinear integral equations, multidimensional renewal theorems, the Laplace transform, large deviation theory for heavy-tailed distributions, auxiliary multitype Bellman-Harris branching processes and other probabilistic-analytic techniques.

This paper completes a series of works devoted to convergence (in a certain sense) of the rescaled front in supercritical CBRW on $\mathbb{Z}^{d}$. It was inspired by the analysis presented in [Carmona, Hu, 2014] for the case of light distribution tails of the random walk jump for $d=1$ and carried out in [Bulinskaya, 2018a; Bulinskaya, 2018b; Bulinskaya, 2019a; Bulinskaya, 2019b; Bulinskaya, 2019c] under various other assumptions. The study reveals that the rate of the population spread depends essentially on the distribution tails of the random walk jump. So, in the case of "light" tails the population front propagates linearly in time (see [Bulinskaya, 2018a] and [Carmona, Hu, 2014]). Whenever the random walk jump distribution is semiexponential, the front moves with power rate, but faster than linearly, see [Bulinskaya, 2019a]. According to [Bulinskaya, 2018b] and [Bulinskaya, 2019c], the assumption of regularly varying tails leads to the exponentially-fast population spread. So far, in the case of regularly varying tails, the problem under consideration has been investigated under the additional assumption of independence of the components of the random walk jump in [Bulinskaya, 2019c]. Contrary to this, we consider an "isotropic" case, when the rate of decay of the jump distribution in different directions is given by the same regularly varying function. In the former case, in the time limit the particles with properly normalized positions concentrate on a random set located at the coordinate axes. As shown in [Bulinskaya, 2019c], for a two-dimensional case, the limiting set forms a cross, and, for any higher dimension $d$, it is a collection of $d$ segments containing the origin. Due to different behavior of jump tails, now the conclusions radically differ from those in [Bulinskaya, 2019c]. Namely, if a set $B$ in $\mathbb{R}^{d}$, containing the origin with its neighborhood, satisfies a certain isotropy condition for just random walk (without branching), then the probability to be eventually captured by $B$ can be found for the population of particles with normalized positions in CBRW and turns out to be positive in general.

There are a number of results on the spread of the population in homogeneous BRW with regularly varying jump distribution tails, see, e.g., [Bhattacharya et al., 2018; Getan et al., 2017], and others. We refer to [Doering, Roberts, 2013; Hu et al., 2012; Molchanov, Yarovaya, 2012; Platonova, Ryadovkin, 2017; Yarovaya, 2017] (see also references therein) for analysis of other aspects of CBRW or its modifications. Most of them are devoted to the long-time behavior of total and local particles numbers. The exception is [Molchanov, Yarovaya, 2012], where the population front of symmetric CBRW with binary splitting and light-tailed increments was defined and studied from the viewpoint of moments boundedness of local particles numbers. Surprisingly, different notions of the propagation front in [Molchanov, Yarovaya, 2012] and [Bulinskaya, 2018a] lead to the same growth rate. However, our approach seems more powerful due to the convergence results under milder restrictions on CBRW. 
The paper is organized as follows. In Section 2 we introduce necessary notation and formulate the main result. In Section 3 we provide its proof. At first we consider the case of a single catalyst and then extend the results obtained to the case of an arbitrary finite number of catalysts.

\section{Model description and main result}

All random elements are defined on a complete probability space $(\Omega, \mathcal{F}, \mathrm{P})$. The index $\mathbf{x}$ in expressions of the form $E_{x}$ and $P_{x}$ marks the starting point of either CBRW or the random walk $S$, depending on the context. Bold font of $\mathbf{x}$ emphasizes that $\mathbf{x}$ is a multidimensional vector, whereas the symbol $x$ means that $x$ is a real number.

Recall the description of CBRW on $\mathbb{Z}^{d}, d \in \mathbb{N}$ (in our setting given in [Bulinskaya, 2018a]). At the initial time $t=0$ there is a single particle that moves on $\mathbb{Z}^{d}$ according to a continuous-time Markov chain $\mathbf{S}=\{\mathbf{S}(t), t \geq 0\}$, generated by the infinitesimal matrix $Q=(q(\mathbf{x}, \mathbf{y}))_{\mathbf{x}, \mathbf{y} \in \mathbb{Z}^{d}}$. Assume that $\mathbf{S}$ is irreducible and space-homogeneous, with the conservative matrix $Q$, i.e., $Q$ has finite elements and

$$
q(\mathbf{x}, \mathbf{y})=q(\mathbf{x}-\mathbf{y}, \mathbf{0}), \quad \sum_{\mathbf{y} \in \mathbb{Z}^{d}} q(\mathbf{x}, \mathbf{y})=0
$$

where $q(\mathbf{x}, \mathbf{y}) \geq 0$ for $\mathbf{x} \neq \mathbf{y}$ and $q:=-q(\mathbf{x}, \mathbf{x}) \in(0, \infty)$, for any $\mathbf{x}, \mathbf{y} \in \mathbb{Z}^{d}$. The requirement of [Molchanov, Yarovaya, 2012] and [Platonova, Ryadovkin, 2017] that $Q$ be symmetric is not imposed.

When this particle hits a finite set of catalysts $W=\left\{\mathbf{w}_{1}, \ldots, \mathbf{w}_{N}\right\} \subset \mathbb{Z}^{d}$, say at the point $\mathbf{w}_{k}$, it spends there random time, distributed exponentially with parameter $\beta_{k}>0$. The particle either splits there or leaves the point $\mathbf{w}_{k}$ with probabilities $\alpha_{k}$ and $1-\alpha_{k}\left(0 \leq \alpha_{k}<1\right)$, respectively. If the particle branches at the point $\mathbf{w}_{k}$, it produces immediately a random nonnegative integer number $\xi_{k}$ of offsprings, located at the same point $\mathbf{w}_{k}$, and dies instantly. Whenever the particle leaves $\mathbf{w}_{k}$, it jumps to the point $\mathbf{y} \neq \mathbf{w}_{k}$ with probability $\left(1-\alpha_{k}\right) q\left(\mathbf{w}_{k}, \mathbf{y}\right) / q$ and resumes its motion governed by the Markov chain S. All the newly born particles are supposed to behave as independent copies of their parent.

Denote by $f_{k}(s):=\mathrm{E} s \xi_{k}, s \in[0,1]$, the probability generating function of $\xi_{k}, k=1, \ldots, N$. Employ the standard assumption of existence of a finite derivative $f_{k}^{\prime}(1)$, that is, the finiteness of $m_{k}:=\mathrm{E} \xi_{k}$, for any $k=1, \ldots, N$. Moreover, in the present paper we assume the validity of the well-known logarithmic moment condition

$$
\mathrm{E} \xi_{k} \ln \left(\xi_{k}+1\right)<\infty, \quad k=1, \ldots, N
$$

Like the classical branching processes (see, e.g., [Vatutin, 2009]), every CBRW can be classified according to [Bulinskaya, 2015a] and [Bulinskaya, 2015b] as supercritical, critical or subcritical depending on the relationship between characteristics $m_{k}, k=1, \ldots, N$, and certain probabilities of finiteness of hitting times under taboo (see, e.g., [Bulinskaya, 2014]). In critical and subcritical regimes the population degenerates locally with probability 1 . Only in the supercritical regime do the mean total and local particles numbers grow exponentially fast as time tends to infinity. The rate of the exponential growth denoted by $\nu$ is traditionally called the Malthusian parameter and in the supercritical regime $\nu>0$. In the sequel, we consider supercritical CBRW only, since in the framework of other regimes the problem of the rate of the population spread seems ill-posed.

Further on, in view of (1) and Theorem 1.2 in [Brémaud, 1999], one can consider the version of the random walk $\mathbf{S}$ such that

$$
\mathbf{S}(t)=\mathbf{x}+\sum_{i=1}^{\Pi(t)} \mathbf{Y}^{i}, \quad t \geq 0,
$$


where $\mathbf{x} \in \mathbb{Z}^{d}$ is a starting point of the random walk, $\Pi=\{\Pi(t), t \geq 0\}$ is a Poisson process with constant intensity $q$ and $\mathbf{Y}^{i}$ is a value of the ith jump of the random walk, $i \in \mathbb{N}$. The random vectors $\mathbf{Y}^{i}=\left(Y_{1}^{i}, \ldots, Y_{d}^{i}\right), i \in \mathbb{N}$, are i.i.d., have distribution $\mathbf{P}\left(\mathbf{Y}^{1}=\mathbf{y}\right)=q(\mathbf{0}, \mathbf{y}) / q, \mathbf{y} \in \mathbb{Z}^{d}, \mathbf{y} \neq \mathbf{0}$ and do not depend on $\Pi(t)$ for any $t \geq 0$.

Our main assumption of isotropic CBRW with regularly varying tails means (see, e.g., [Borovkov, Borovkov, 2008, Ch. 9, Sec. 3]) that, for a set $A \subset \mathbb{R}^{d}$ separated from the origin by a ball of a radius $b>0$, one has

$$
\mathrm{P}\left(\mathbf{Y}^{1} \in u A\right) \sim V(u) F(A), \quad \text { as } \quad u \rightarrow \infty,
$$

where $V(u)=u^{-\gamma} L_{1}(u), u \geq 0$, is a regularly varying function of index $-\gamma, \gamma \in(0,+\infty), L_{1}(u)$, $u \geq 0$, is a slowly varying function at infinity and $F(A)$ is a functional defined on a suitable class of sets. This functional, the set $A$ and the vector $\mathbf{Y}^{1}$ are such that

$$
\mathrm{P}\left(\mathbf{Y}^{1} \in \mathbf{s}+u A\right) \sim \mathrm{P}\left(\mathbf{Y}^{1} \in u A\right) \quad \text { for } \quad\|\mathbf{s}\|=o(u), \quad u \rightarrow \infty,
$$

where the norm $\|\cdot\|$ in $\mathbb{R}^{d}$ may be chosen arbitrarily. The latter property simply expresses the continuity of the functional $F$ : we have $F(\mathbf{v}+A) \sim F(A)$, as $\|\mathbf{v}\| \rightarrow 0$. Observe that the complement of the set $A$ is $B:=A^{c} \subset \mathbb{R}^{d}$ containing the origin together with the ball of radius $b>0$. According to [Seneta, 1976, Ch. 1, Sec. 5], property $5^{\circ}$, there exists an asymptotically uniquely determined inverse function $V^{-1}(u), u \geq 0$, in the sense that $1 / V\left(V^{-1}(u)\right) \sim u, V^{-1}(1 / V(u)) \sim u$, as $u \rightarrow \infty$, and $V^{-1}(u)=u^{1 / \gamma} L_{2}(u), u \geq 0$, where $L_{2}$ is a slowly varying function at infinity.

Let $Z(t)$ be the (random) set of particles existing in CBRW at time $t \geq 0$. For a particle $z \in Z(t)$, denote by $\mathbf{X}^{z}(t)=\left(X_{1}^{z}(t), \ldots, X_{d}^{z}(t)\right)$ its position at time $t$. To study the rate of the spread of particles population on $\mathbb{Z}^{d}$, we divide their coordinates by a specified normalizing factor. The proper choice of the factor leads to existence of a nontrivial limit of the scaled positions of particles "at the front" at time $t$ as $t \rightarrow \infty$. Our normalizing factor is

$$
N(t):=V^{-1}\left(e^{\nu t}\right)=e^{\nu t / \gamma} L_{2}\left(e^{\nu t}\right), \quad t \geq 0 .
$$

We also assume that the normalizing factor $N(t), t \geq 0$, belongs to the maximum jump approximation zone (see, e.g., [Borovkov, Borovkov, 2008, p. 251]) of the random walk S, i.e.,

$$
\mathrm{P}(\mathbf{S}(u) / N(t) \in A) \sim q u \mathrm{P}\left(\mathbf{Y}^{1} \in N(t) A\right), \quad t \rightarrow \infty,
$$

uniformly in $u / t \in[0,1]$. Broad sufficient conditions for the validity of (4) can be found, e.g., in Theorem 9.3.1 of [Borovkov, Borovkov, 2008].

Theorem 1. Let assumptions (1) and (2) be satisfied for supercritical CBRW on $\mathbb{Z}^{d}$ with Malthusian parameter $\nu$. Let also conditions (3) and (4) be valid for a set $A \subset \mathbb{R}^{d}$, separated from the origin by a ball of a radius $b>0$. Then there exists a function $\varphi(\lambda ; \mathbf{x}), \lambda \geq 0, \mathbf{x} \in \mathbb{Z}^{d}$, such that, for any $\mathrm{x} \in \mathbb{Z}^{d}$, one has

$$
\mathrm{P}_{\mathbf{x}}\left(\forall z \in Z(t): \mathbf{X}^{z}(t) / N(t) \notin A\right) \rightarrow \varphi(F(A) ; \mathbf{x}), \quad t \rightarrow \infty .
$$

Here $\varphi(\lambda ; \mathbf{x}) \in(0,1), \varphi(0 ; \mathbf{x})=1$, and $\varphi(\lambda ; \mathbf{x})$ tends to the local extinction probability of the population in $C B R W$ as $\lambda \rightarrow \infty$, for each fixed $\mathbf{x} \in \mathbb{Z}^{d}$. The function $\varphi(\cdot ; \mathbf{x})$ satisfies the system of nonlinear integral equations appearing in (5) of [Bulinskaya, 2018b] and its solution is unique in a certain function class (see Theorem 1 in [Bulinskaya, 2018b]).

Invoking different sets $A$ under specific assumptions on the common distribution of the components of $\mathbf{Y}^{1}$ leads to a more detailed description for the limiting shape of the front of the particles population. 


\section{Proof}

Consider CBRW with a single catalyst $\mathbf{w}_{1}$ located, without loss of generality, at the origin, that is, $W=\left\{\mathbf{w}_{1}\right\}$ with $\mathbf{w}_{1}=\mathbf{0}$, and the starting point being $\mathbf{0}$ as well.

Let $E(t ; U):=\mathrm{P}_{0}\left(\exists z \in Z(t): \mathbf{X}^{z}(t) \in U\right)$, for a set $U \subset \mathbb{R}^{d}$. The following lemma (proved in [Bulinskaya, 2018b] for $d=1$ and in [Bulinskaya, 2019a] for $d>1$ ) provides an integral equation for the probability $E(t ; U)$.

Lemma 1. Let condition (1) be valid. Then the probability $E(t ; U), t \geq 0, U \subset \mathbb{R}^{d}, \mathbf{0} \notin U$, satisfies the nonlinear integral equation of convolution type

$$
E(t ; U)=\alpha_{1} \int_{0}^{t}\left(1-f_{1}(1-E(t-s ; U))\right) d G_{1}(s)+\left(1-\alpha_{1}\right) \int_{0}^{t} E(t-s ; U) d G_{1,1}(s)+I(t ; U),
$$

where

$$
\begin{aligned}
\frac{q I(t ; U)}{\left(1-\alpha_{1}\right) \beta_{1}}:=\mathrm{P}_{\mathbf{0}}(\mathbf{S}(t) \in U)-\int_{0}^{t} \mathrm{P}_{\mathbf{0}}(\mathbf{S}(t-s) \in U) d F_{\mathbf{0}, \mathbf{0}}(s) \\
\\
-\frac{\beta_{1}-q}{\beta_{1}} \int_{0}^{t} \mathrm{P}_{\mathbf{0}}(\mathbf{S}(t-s) \in U) d\left(G_{1}(s)-G_{1} * F_{\mathbf{0}, 0}(s)\right) .
\end{aligned}
$$

Here the function $G_{1}(t):=1-e^{-\beta_{1} t}, t \geq 0 . F_{\mathbf{0}, 0}(t), t \geq 0$, is a cumulative distribution function (c.d.f.) of the first hitting time of the point $\mathbf{0}$ by the random walk $\mathbf{S}$, when the starting point of $\mathbf{S}$ is $\mathbf{0}$. Similarly, $\bar{F}_{\mathbf{0}, \mathbf{0}}(t), t \geq 0$, is a c.d.f. of the first hitting time of the point $\mathbf{0}$ by the random walk $\mathbf{S}$ after exiting the starting point $\mathbf{0}$. The symbol $*$ stands for the convolution operation and $G_{1,1}(t):=G_{1} * \bar{F}_{\mathbf{0}, \mathbf{0}}(t), t \geq 0$.

The definition of the supercritical regime of CBRW (see [Bulinskaya, 2015a]) implies that $\alpha_{1} m_{1}+\left(1-\alpha_{1}\right) F_{\mathbf{0}, \mathbf{0}}(\infty)>1$ and $\alpha_{1} m_{1} G_{1}^{*}(\nu)+\left(1-\alpha_{1}\right) G_{1}^{*}(\nu) \bar{F}_{\mathbf{0 , 0}}^{*}(\nu)=1$. In terms of the function $G(t):=\alpha_{1} m_{1} G_{1}(t)+\left(1-\alpha_{1}\right) G_{1} * \bar{F}_{\mathbf{0}, \mathbf{0}}(t), t \geq 0$, it means that $G^{*}(\nu)=1$. Here $H^{*}(\lambda), \lambda \geq 0$, denotes the Laplace transform of a c.d.f. $H(t), t \geq 0$, with support on nonnegative semiaxis, i.e., $H^{*}(\lambda):=\int_{0-}^{\infty} e^{-\lambda t} d H(t)$.

Lemma 2. Let assumptions (1) and (2) be satisfied for supercritical CBRW on $\mathbb{Z}^{d}$ with a single catalyst $\mathbf{w}_{1}=\mathbf{0}$ and Malthusian parameter $\nu$. Let also conditions (3) and (4) be valid for a set $A \subset \mathbb{R}^{d}$, bounded from the origin by a ball of a radius $b>0$. Then one has

$$
\mathrm{P}_{\mathbf{0}}\left(\forall z \in Z(t): \mathbf{X}^{z}(t) / N(t) \notin A\right) \rightarrow \varphi(F(A) ; \mathbf{0}), \quad t \rightarrow \infty .
$$

The function $\varphi(\lambda ; \mathbf{x}), \lambda \geq 0, \mathbf{x} \in \mathbb{Z}^{d}$, appears in Theorem 1 .

Proof. The proof of Lemma 2 is similar to analysis in [Bulinskaya, 2018b] of the long-time behavior of the maximum of $\mathrm{CBRW}$ on $\mathbb{Z}$ with regularly varying tails. The key differences are the following two. 1) Here we study the asymptotic behavior of the probability $\mathrm{P}_{\mathbf{0}}\left(\exists z \in Z(t): \mathbf{X}^{z}(t) / N(t) \in A\right)$, for a set $A \subset \mathbb{R}^{d}$, instead of $\mathrm{P}_{\mathbf{0}}\left(\exists z \in Z(t): X^{z}(t) / N(t) \geq \lambda\right)$, for $\lambda>0.2)$ Assumption (4) implies that

$$
\mathrm{P}_{\mathbf{0}}(\mathbf{S}(t-s) / N(t) \in A) \sim q(t-s) V(N(t)) F(A) \sim q(t-s) e^{-\nu t} F(A),
$$

as $t \rightarrow \infty$, uniformly in $s / t \in[0,1]$. The last relation differs from its one-dimensional counterpart by the factor $F(A)$. Within the study of the maximum of CBRW on $\mathbb{Z}$ in [Bulinskaya, 2018b], the corresponding factor is just $\lambda$. The rest of the proof of Lemma 2 repeats the proofs of Lemmas 3-6 and Lemma 8 in [Bulinskaya, 2018b] and is thus omitted. 
Whenever the catalysts set $W$ consists of $\mathbf{w}_{1}, \ldots, \mathbf{w}_{N} \in \mathbb{Z}^{d}$ and $N>1$, the proof of Theorem 1 follows the proof scheme of Lemmas 1 and 2. However, now we have to consider several probabilities $E_{\mathbf{w}_{j}}(t ; U):=\mathrm{P}_{\mathbf{w}_{i}}\left(\exists z \in Z(t): \mathbf{X}^{z}(t) \in U\right), j=1, \ldots, N$, instead of just $E(t ; U)$, as in Lemma 1. Correspondingly, equation (5) in Lemma 1 has to be replaced by a system of equations, see Step 4 of the proofs in [Bulinskaya, 2019a]. The function $G(t)$ transforms into a matrix $\left(G_{i, j}^{(N)}(t)\right)_{i, j=1}^{N}, t \geq 0$, with entries $G_{i, j}^{(N)}(t):=\delta_{i, j} \alpha_{i} m_{i} G_{i}(t)+\left(1-\alpha_{i}\right) G_{i} * W_{j} \bar{F}_{\mathbf{w}_{i}, \mathbf{w}_{j}}(t)$. Here $\delta_{i, j}$ is the Kronecker delta, $G_{i}(t):=1-e^{-\beta_{i} t}, W_{j} \bar{F}_{\mathbf{w}_{i}, \mathbf{w}_{j}}(t), t \geq 0$, is a c.d.f. of the first hitting time of the point $\mathbf{w}_{j}$ (by the random walk $\mathbf{S}$ ) after exiting the starting point $\mathbf{w}_{i}$ under taboo $W_{j}:=W \backslash\left\{\mathbf{w}_{j}\right\}$ (see, e.g., [Bulinskaya, 2014]). Then we use multidimensional renewal theory in the framework of [Crump, 1970] and analyze the time limit of the solution of the system of nonlinear integral equations employing the approach in [Kaplan, 1975]. The details can be recovered similarly to the proof of Theorem 1 in [Bulinskaya, 2018b] and the proofs (Step 4) in [Bulinskaya, 2019a]. Thus, the claim of Theorem 1 is established.

\section{References}

Bhattacharya A., Hazra R.S., Roy P. Branching random walks, stable point processes and regular variation // Stochastic Processes and their Applications. - 2018. - Vol. 128, No. 1. - P. 182-210.

Borovkov A.A., Borovkov K. A. Asymptotic Analysis of Random Walks. Heavy-tailed distributions. Cambridge: Cambridge University Press, 2008. - 625 p.

Brémaud P. Markov chains: Gibbs Fields, Monte-Carlo Simulation, and Queues. - New York: Springer, 1999. - $444 \mathrm{p}$.

Bulinskaya E. Vl. Finiteness of hitting times under taboo // Statistics and Probability Letters. - 2014. Vol. 85, No. 1. - P. 15-19.

Bulinskaya E. Vl. Complete classification of catalytic branching processes // Theory of Probability and its Applications. - 2015. - Vol. 59, No. 4. - P. 545-566.

Bulinskaya E. Vl. Strong and weak convergence of the population size in a supercritical catalytic branching process // Doklady Mathematics. - 2015. - Vol. 92, No. 3. - P. 714-718.

Bulinskaya E. Vl. Spread of a catalytic branching random walk on a multidimensional lattice // Stochastic Processes and their Applications. - 2018. - Vol. 128, No. 7. - P. 2325-2340.

Bulinskaya E. Vl. Maximum of catalytic branching random walk with regularly varying tails // ArXiv:1808.01465. - 2018. - P. 1-17.

Bulinskaya E.Vl. Catalytic branching random walk with semiexponential increments // ArXiv:1902.00994. - 2019. - P. 1-20.

Bulinskaya E. Vl. Maximum of a catalytic branching random walk // Russian Mathematical Surveys. 2019. - Vol. 74, No. 3. - P. 546-548.

Bulinskaya E. Vl. Multidimensional catalytic branching random walk with regularly varying tails // Proceedings of the 2019 2nd International Conference on Mathematics and Statistics. Prague, Czech Republic. - July 08-10, 2019. - ACM International Conference Proceeding Series. New York, 2019. - P. 6-13.

Carmona Ph., Hи Y. The spread of a catalytic branching random walk // Annales de l'Institut Henri Poincaré (B). Probabilités at Statistiques. - 2014. - Vol. 50, No. 2. - P. 327-351.

Crump K.S. On systems of renewal equations // Journal of Mathematical Analysis and its Applications. - 1970. - Vol. 30, No. 2. - P. 425-434.

Doering L., Roberts M. Catalytic branching processes via spine techniques and renewal theory // Lecture Notes in Mathematics: Séminaire de Probabilités XLV. - Vol. 2078. - Cham: Springer, 2013. - P. 305-322. 
Getan A., Molchanov S., Vainberg B. Intermittency for branching walks with heavy tails // Stochastics and Dynamics. - 2017. - Vol. 17, No. 6. - P. 1750044.

Hu Y., Topchii V.A., Vatutin V.A. Branching random walk in $\mathbf{Z}^{4}$ with branching at the origin only // Theory of Probability and its Applications. - 2017. - Vol. 56, No. 2. - P. 193-212.

Kaplan $N$. The supercritical multitype age-dependent branching process // Journal of Mathematical Analysis and its Applications. - 1975. - Vol. 50, No. 1. - P. 164-182.

Molchanov S. A., Yarovaya E. B. Branching processes with lattice spatial dynamics and a finite set of particle generation centers // Doklady Mathematics. - 2012. - Vol. 446, No. 3. - P. 259-262.

Platonova M. V., Ryadovkin K.S. On the mean number of particles of a branching random walk on $\mathbb{Z}^{d}$ with periodic sources of branching // Doklady Mathematics. - 2018. - Vol. 97, No. 2. P. $140-143$.

Seneta E. Regularly Varying Functions. - Heidelberg: Springer, 1976. - 112 p.

Shi Z. Branching Random Walks. - Cham: Springer, 2015. - 133 p.

Vatutin V.A. Bellman-Harris Branching Processes. - Moscow: MIRAS, 2009. - 111 p. (in Russian).

Yarovaya E. B. Positive discrete spectrum of the evolutionary operator of supercritical branching walks with heavy tails // Methodology and Computing in Applied Probability. - 2017. - Vol. 19, No. 4. - P. 1151-1167. 
